\title{
Sensitivity of latex agglutination faecal occult blood test in the Florence District population-based colorectal cancer screening
}

\section{programme}

\author{
G Castiglione ${ }^{*, 1}$, CB Visioli' ${ }^{2}$ S Ciatto', G Grazzini ${ }^{3}$, AG Bonanomi', T Rubeca ${ }^{4}$, P Mantellini ${ }^{3}$ and M Zappa ${ }^{2}$ \\ 'Diagnostic Imaging Unit, CSPO, Viale A, Volta 171, Florence 50 I31, Italy; ' Epidemiology Unit, CSPO Via di San Salvi I2, Florence 50135, Italy; \\ ${ }^{3}$ Screening Unit, CSPO Viale A, Volta I7I, Florence 50 I3I, Italy; ${ }^{4}$ Laboratory Unit, CSPO Via Cosimo il Vecchio 2, Florence 50 I39, Italy
}

\begin{abstract}
We evaluated the sensitivity for colorectal cancer (CRC) of the latex agglutination test (LAT), an immunochemical test routinely used in the Florence District screening programme since 2000. Sensitivity was calculated by the proportional interval cancer incidence method in a population of 27503 consecutive subjects screened in 2000-2002, interval cancers being identified by linkage to the Tuscany Cancer Registry files. Sensitivity was calculated overall and by gender, age, time since last negative LAT, CRC site, and rank of screening. Overall I- and 2-year sensitivity estimates were 80.7 and $71.5 \%$, respectively, suggesting that faecal occult blood testing screening sensitivity may be suboptimal due to testing or programme quality problems. Increasing screening sensitivity might be achieved if the detection rate of advanced adenomas could be increased without unacceptable loss in specificity.

British Journal of Cancer (2007) 96, 1750- 1754. doi: 10.1038/sj.bjc.6603759 www.bjcancer.com

Published online 24 April 2007

(c) 2007 Cancer Research UK
\end{abstract}

Keywords: colorectal cancer; screening; faecal occult blood tests; sensitivity; proportional incidence method

Screening for colorectal cancer (CRC) by faecal occult blood testing (FOBT) has been effective in reducing CRC mortality and incidence (Towler et al, 2000), and is recommended in several countries as current health policy. Randomised trials demonstrating FOBT screening effectiveness used a 3-day guaiac-based test (G-FOBT), a chemical test based on haemoglobin peroxidase-like activity whose accuracy is affected by dietary factors such as the presence of nonhuman haemoglobin and peroxidases in vegetables, or by certain drugs, particularly NSAIDs. Sensitivity of G-FOBT for CRC has been reported to be as low as $43-66 \%$, based on a 2-year screening interval (Jensen et al, 1992; Launoy et al, 1997; Moss et al, 1999; Jouve et al, 2001); attempts to increase sensitivity either by rehydration (Church et al, 1997) or by increased reagent concentration (Petrelli et al, 1994; Allison et al, 1996) were associated with unacceptable loss in specificity. Several studies have suggested that immunochemical FOBT (I-FOBT) is more sensitive and specific than G-FOBT (Castiglione et al, 1997; Saito et al, 2000; Zappa et al, 2001; Levi et al, 2006; Guittet et al, 2007), requiring no dietary restrictions, and might substantially improve screening cost effectiveness (Castiglione et al, 1997; Saito et al, 2000). Further progress in I-FOBT use was made by the introduction of the latex agglutination test (LAT), a quantitative and fully automated test (Yamamoto et al, 1990), which made it possible to choose the positivity cutoff values to optimise the balance between sensitivity and specificity, though the debate about the optimal cutoff point continues (Itoh et al, 1996;

*Correspondence: Dr G Castiglione; E-mail: g.castiglione@cspo.it Received 18 January 2007; revised 19 March 2007; accepted 30 March 2007; published online 24 April 2007
Castiglione et al, 2000; Nakama et al, 2001; Edwards, 2005; Vilkin et al, 2005). Sensitivity of G-FOBT and a previously used I-FOBT (reversed passive haemagglutination (RPHA)) for CRC, based on proportional interval cancer incidence, was determined previously (Zappa et al, 2001). In the present study we have evaluated LAT sensitivity using similar methodology.

\section{POPULATION AND METHODS}

\section{Setting}

A population-based FOBT screening programme has existed in Florence District since 1982, run by the Istituto Scientifico per la Prevenzione Oncologica (CPSO), its efficacy in reducing CRC mortality supported by a case-control study (Zappa et al, 1997). Reversed passive haemagglutination was used to replace G-FOBTin 1995 (Castiglione et al, 1997). A further study (Castiglione et al, 2000) showed that LAT (OC-Hemodia, developed with the OCSensor instrument, Eiken, Tokyo, Japan, and henceforth referred to as LAT) had a performance comparable with RPHA, and it was adopted as the standard test in our programme in January 2000, with a positivity threshold of $100 \mathrm{ng} \mathrm{Hb} \mathrm{ml}^{-1}$ of sample solution. The main features and protocol have been reported in detail (Grazzini et al, 2004). Colonoscopy was recommended to FOBTpositive subjects.

\section{Population}

Subjects aged 50-70, living in 19 municipalities in the Province of Florence, and attending FOBT screening from January 2000 to December 2002 were eligible for the present study. 
Incident CRCs in 2000-2003 in Florence District residents were identified by linking the Tuscany Cancer Registry (http:// www.cspo.it/REGISTRI//REGISTRO_RTT/rapporti/index.html) (by name, date, and place of birth), with screening archives. In case of partial matching (i.e. name and date of birth, not place of birth), manual assessment of records was performed. All CRCs diagnosed within 2 years after (1) a negative-LAT test or (2) a positive screen followed by a negative assessment, were regarded as interval cancers. Only interval cancers following a negative LAT were used to calculate 'screening test sensitivity', whereas both classes of interval cancers were used to assess 'screening programme sensitivity'. In this way, the limits of the test were distinguished from those of the programme and the present figures compared with similar estimates in the literature.

In the sensitivity assessment, LAT-positive (LAT +$)$ cases who refused assessment but had CRC diagnosed within 1 year since LAT were regarded as being detected by the programme, whereas cases with CRC diagnosed after 1 year were excluded. Screen detected and interval CRCs were classified according to bowel subsite and Dukes staging.

\section{Statistical analysis}

Sensitivity was estimated by the proportional interval cancer incidence method (Day, 1985) which compares interval cancers within a given interval following a negative screening, or a positive test followed by a negative assessment, with the expected cancer incidence in the absence of screening (underlying incidence) according to the formula:

$$
\text { sensitivity }=1-(\mathrm{I}(\mathrm{t}) / \mathrm{I})
$$

where $\mathrm{I}(\mathrm{t})$ is the observed interval cancers during interval $t$ and $\mathrm{I}$ is the underlying incidence. Person-years at risk were calculated from the date of the first negative LAT. Observation ended (a) at 2 years, or (b) at interval cancer occurrence, or (c) at death from any cause, or (d) at the end of the study (December 2003), or (e) at the date of subsequent LAT if performed after an interval shorter than 2 years. Underlying CRC incidence was calculated using personyears and age-/sex-specific incidence rates provided by the Tuscany Cancer Registry (Table 1). Sensitivity was estimated both overall and by gender, age (50-59 or 60-70) at LAT, time since last negative LAT (first or second year of the interval), cancer location (rectum and rectosigmoid junction: $\mathrm{ICDO}=154$; colon: ICDO $=153$ ), and rank of screening (first or subsequent). Confidence intervals (95\% CI) were calculated on the basis of the exact Poisson's distribution, while statistical differences among strata (i.e. gender, age, site, time since last test, rank of screening) were tested by Gaussian approximation of the log likelihood (Clayton and Hills, 1993). Rate ratios (RRs) among strata were computed.

\section{RESULTS}

From January 2000 to December 2002, 24913 subjects performed 27503 LATs, attendance rates in our programme being about $50 \%$ in the study period. Age/sex distribution of subjects at the time of the LAT are shown in Table 2 . Table 3 presents LAT positivity and detection rates of colorectal lesions. Overall, LAT was positive in 1097 of 27503 attending subjects (4.0\%). Further diagnostic workup was refused by $138(12.6 \%)$ and accepted by 959 of 1097 LAT + subjects. Colonoscopy was complete in 788 of the $959(82.2 \%)$ and was incomplete in the remaining $(17.3 \%)$. Double contrast barium enema was performed with incomplete colonoscopy in 106 of $166(63.9 \%)$ subjects, and alone in five of 959 LAT + cases $(0.5 \%)$ not complying with colonoscopy and referred to their family physicians for diagnostic work up.
Table I Incidence rates (per 100.000 person-years) of colorectal cancer in the Florence District by sex, age, and subsite in the period 1997-2002: Tuscany Cancer Registry

\begin{tabular}{lrrr}
\hline Age (years) & Colon & Rectum & Overall \\
\hline Males & & & \\
$50-59$ & 59.6 & 40.2 & 99.8 \\
$60-69$ & 146.3 & 75.7 & 222.0 \\
$70-74$ & 241.6 & 115.1 & 356.7 \\
Females & & & \\
$50-59$ & 42.9 & 21.0 & 63.9 \\
$60-69$ & 87.6 & 39.6 & 127.2 \\
$70-74$ & 135.6 & 56.4 & 192.0 \\
\hline
\end{tabular}

Table 2 Age and sex distribution of subjects at the time they performed latex agglutination test

\begin{tabular}{|c|c|c|c|c|c|c|}
\hline \multirow[b]{2}{*}{ Age (years) } & \multicolumn{2}{|c|}{ Males } & \multicolumn{2}{|c|}{ Females } & \multicolumn{2}{|c|}{ Total } \\
\hline & $N$ & $\%$ & $N$ & $\%$ & $N$ & $\%$ \\
\hline $50-59$ & 6,580 & 50.0 & 7,198 & 50.1 & 13,778 & 50.1 \\
\hline $60-69$ & 6,134 & 46.7 & 6,677 & 46.5 & $|2,8| \mid$ & 46.6 \\
\hline 70 & 431 & 3.3 & 483 & 3.4 & 914 & 3.3 \\
\hline Total & 13,145 & 100.0 & 14,358 & 100.0 & 27,503 & 100.0 \\
\hline
\end{tabular}

Table 3 Colorectal lesions detected of Latex Agglutination Test

\begin{tabular}{|c|c|c|c|}
\hline & No. & Rate & $95 \% \mathrm{Cl}$ \\
\hline Screening tests & 27503 & & \\
\hline Positive tests & 1097 & $4.0 \%$ & $3.8-4.2$ \\
\hline $\begin{array}{l}\text { Screen positive subjects refusing } \\
\text { assessment }\end{array}$ & 138 & $12.6 \%$ & \\
\hline Screen-detected cancers & 65 & $2.36 \%$ & $1.8-3.0$ \\
\hline $\begin{array}{l}\text { No. screen-detected advanced } \\
\text { adenomas }\end{array}$ & 219 & $7.96 \%$ & $6.95-9.08$ \\
\hline $\begin{array}{l}\text { No. screen-detected cancer and } \\
\text { advanced adenomas }\end{array}$ & 284 & $10.3 \%$ & $9.16-11.59$ \\
\hline \multicolumn{4}{|l|}{ Interval cancers within 2 years } \\
\hline I After a negative test & 16 & $0.58 \%$ & $0.33-0.94$ \\
\hline 2 After a negative test or a & 18 & $0.65 \%$ & $0.39-1.03$ \\
\hline $\begin{array}{l}\text { positive test followed by negative } \\
\text { assessment }\end{array}$ & & & \\
\hline
\end{tabular}

A total of 65 cancers were detected at screening, a detection rate of 2.4\%. Screen-detected CRCs' Dukes stage was A, B, C, and D in $30,16,11$, and six subjects respectively; two screen-detected neoplasms were carcinoids. Two cancers detected within 1 year from a positive-LAT case who had refused colonoscopy in our reference centres were regarded as screen-detected cancers. Advanced adenoma(s) (larger than $9 \mathrm{~mm}$, or with high-grade dysplasia, or with villous component $\geqslant 20 \%$ ) was detected in 219 subjects.

For calculation of programme sensitivity 136 FOBT + subjects refusing assessment were excluded; two of them had CRC detected after 1 year, and within 2 years of testing. Overall, 16 interval cancers were detected in the 2 years following a negative LAT. Dukes stage was A, B, C, and D in 3, 4, 3, and 4, respectively and was missing in two cases. Furthermore two interval cancers were detected within 2 years of a positive LAT followed by a negative assessment (1 Dukes B and 1 Dukes D). 
Table 4 Latex test sensitivity

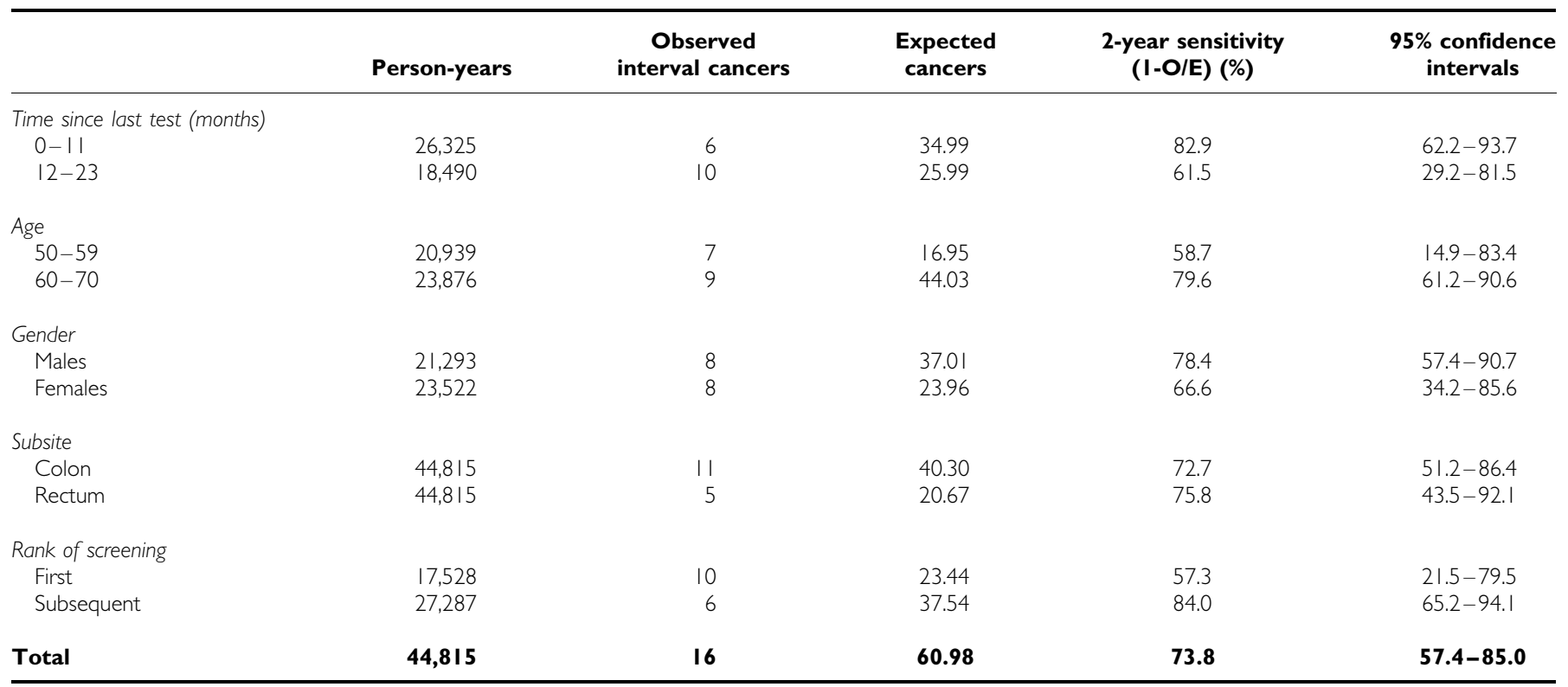

Person-years, observed interval cancers, expected colorectal cancers and screening sensitivity by time since last test, age groups, gender, site, and rank of screening, according to a 2-year period of observation following a negative latex test.

Table 5 Programme sensitivity

\begin{tabular}{|c|c|c|c|c|c|}
\hline & Person-years & $\begin{array}{l}\text { Observed interval } \\
\text { cancers }\end{array}$ & $\begin{array}{l}\text { Expected } \\
\text { cancers }\end{array}$ & $\begin{array}{l}\text { 2-year sensitivity } \\
\text { (I-O/E) (\%) }\end{array}$ & $\begin{array}{l}95 \% \text { confidence } \\
\text { intervals }\end{array}$ \\
\hline \multicolumn{6}{|c|}{ Time since last test (months) } \\
\hline $12-23$ & 19,127 & 11 & 26.96 & 59.2 & $27.0-79.6$ \\
\hline \multicolumn{6}{|l|}{ Age } \\
\hline $50-59$ & 21,552 & 8 & 17.45 & 54.2 & $9.7-80.2$ \\
\hline \multicolumn{6}{|l|}{ Gender } \\
\hline Males & 22,108 & 8 & 38.52 & 79.2 & $59.1-91.0$ \\
\hline Females & 24,239 & 10 & 24.72 & 59.6 & $25.6-80.6$ \\
\hline \multicolumn{6}{|l|}{ Site } \\
\hline Colon & 46,347 & 13 & 41.80 & 68.9 & $46.8-83.4$ \\
\hline Rectum & 46,347 & 5 & 21.44 & 76.7 & $45.6-92.4$ \\
\hline Total & 46,347 & 18 & 63.24 & 71.5 & $55.0-83.1$ \\
\hline
\end{tabular}

Person-years, observed interval cancers, expected colorectal cancers and screening sensitivity by time since last test, age groups, gender, subsite, and rank of screening, with a 2 year period of observation following a negative latex test or a positive-latex test followed by a negative assessment.

Table 4 presents screening test sensitivity, person-years, expected numbers of cancers and sensitivity estimates overall and by time since last LAT, age, gender, location in the large bowel, or rank of screening. Overall, 2-year sensitivity was $73.8 \%$ (CI 57.4-85.0). Although no statistically significant difference was observed between sensitivity estimates (probably due to the small sample size), sensitivity was higher for subsequent ranks than at first screening $(84.0$ vs $57.3 \%$, respectively, $\mathrm{RR}=2.27,95 \%$ $\mathrm{CI}=0.97-7.34)$ and for elderly compared to younger subjects (79.5 vs $58.7 \%$, respectively, $\mathrm{RR}=2.02,95 \% \mathrm{CI}=0.75-5.42$ ). As expected, sensitivity in the first year was higher than that in the second year $(82.9$ vs $61.5 \%$, respectively, $\mathrm{RR}=0.45 ; 95 \%$ $\mathrm{CI}=0.16-1.23)$. No difference was evident by gender or site.
Overall, 2-year programme sensitivity was $71.5 \%$ (CI $55.0-83.1$ ), as shown in Table 5. No significant difference was evident by age, gender, site, or rank. Also, the difference in sensitivity between the first and second year (80.7 vs 59.2\%, respectively, $\mathrm{RR}=0.47,95 \%$ $\mathrm{CI}=0.18-1.22)$ was not significant.

\section{DISCUSSION}

The present study was based on a relatively large series with cancer registry follow-up, allowing a reliable estimate of sensitivity according to the proportional interval cancer incidence method, probably the most reliable available. The traditional method 
(sensitivity $=$ screen detected cancers/screen detected cancers + interval cancers) is open to criticism, as cancers with a long sojourn time and unlikely to surface clinically within 2 years, may be included among screen-detected cancers, particularly at prevalent screen, causing a lead time bias with overestimation of sensitivity. In fact, if we had used the traditional method the 1-year programme sensitivity would have been $91.6 \%(65 / 65+6)$ compared to our $82.9 \%$, and the 2-year sensitivity $80.3 \%$ (65/ $65+16)$ compared to our $71.5 \%$.

Nevertheless, our sensitivity method may be subject to selection bias. In fact, the method compares the incidence of interval CRC in compliers with the underlying incidence expected in the general population, assuming similar CRC incidence in compliers and noncompliers. Should this be greater in compliers, sensitivity in our study would be underestimated (Zappa et al, 1998). Unfortunately, we have been unable to get relevant information, and a study is in progress to verify the magnitude of any such bias by measuring the observed/expected CRC incidence ratio among noncompliers.

We regarded FOBT + subjects who refused assessment but were diagnosed with CRC within 1 year of the positive screen as screen detected: in fact delayed assessment may still be motivated by (and ascribed to) the positive screen, but becomes unlikely after 1 year, whereas the probability of symptomatic CRC being diagnosed increases. The latter cases were therefore excluded when assessing programme sensitivity.

The sample size was limited, which may have prevented statistically significant results for age, gender, and bowel site analyses. Similarly, lack of statistical significance for sensitivity estimates based on time since last negative test or rank of screening may be due to the small number of events.

Although several studies have compared I-FOBT and G-FOBT (Petrelli et al, 1994; Allison et al, 1996; Saito et al, 2000), few have reported sensitivity estimates based on interval CRCs: Nakama et al (1996) reported values by the traditional method, of $90.9,83.3$, and $71.4 \%$ within 1,2 , and 3 years, respectively, using the 1 -day Monohaem, immunological test. Zappa et al (2001) used the proportional interval cancer incidence method, and reported 1and 2-year sensitivity estimates for CRC for 1-day RPHA testing of 89 and $82 \%$, respectively, whereas corresponding estimates for 3 -day G-FOBT were 64 and 50\%, respectively. Using the traditional method, Launoy et al (2005) reported a 2-year sensitivity estimate of $85 \%$ for a RPHA-derived test (Magstream) with a low-positivity cutoff and a recall rate of $6 \%$.

Latex agglutination test sensitivity in our study is higher than currently reported for unhydrated G-FOBT and slightly lower than in our previous study employing this method (Zappa et al, 2001) using RPHA I-FOBT as a screening test $(80.0$ vs $89 \%$ at 1 -year and 71.5 vs $82.5 \%$ at 2-year interval, respectively) or in Launoy's study based on a similar automated analytical method. These results may reflect differences in analytical or statistical methods.

\section{REFERENCES}

Allison JE, Tekama IS, Ransom LJ, Adrian AL (1996) A comparison of fecal occult-blood tests for colorectal-cancer screening. N Engl J Med 334: $155-159$

Castiglione G, Zappa M, Grazzini G, Sani C, Mazzotta A, Mantellini P, Ciatto $S$ (1997) Cost analysis in a population based screening programme for colorectal cancer: comparison of immunochemical and guaiac faecal occult blood testing. J Med Screen 4: 142-146

Castiglione G, Zappa M., Grazzini G, Rubeca T, Turco P, Sani C, Ciatto S (2000) Screening for colorectal cancer by faecal occult blood test: comparison of immunochemical tests. J Med Screen 7: 35-37

Church TR, Ederer F, Mandel JS (1997) Fecal occult blood screening in the Minnesota study: sensitivity of the screening test. J Natl Cancer Inst 89: $1440-1448$
Other studies have assessed I-FOBT sensitivity using colonoscopy as the gold standard: Yoshinaga et al (1995) determined LAT sensitivity for CRC in 855 subjects undergoing colonoscopy; in 23 detected CRC, sensitivity estimates were 90 and $100 \%$ for Dukes A and B + stages, respectively. Recently, Vilkin et al (2005) reported LAT colonoscopy-based sensitivity estimates of 66.7 and $100 \%$ with 1- and 3-day sampling, respectively. A large colonoscopybased study of 1-day I-FOBT (Magstream) (Morikawa et al 2005) reported an overall sensitivity of $66 \%$, estimates being stage (advanced CRC > early CRC) and site dependent (left colon > right colon). Studies comparing FOBT results with colonoscopy for assessing FOBT sensitivity are not comparable with our study as they are open to lead time or overdiagnosis biases.

One possible factor negatively affecting screening programme sensitivity could be the suboptimal quality of the assessment phase. In our study two interval cancers were detected in FOBT + subjects after a negative assessment, so even though we are not sure that CRC was present at the time of testing, these findings probably indicate weaknesses in our programme in the recall and assessment phases. Its sensitivity might be improved by referring FOBT + subjects to selected centres with high endoscopy standards, to minimise the false-negative assessments, and by investing resources to improve compliance.

With respect to the screening test, annual rather than biennial testing would probably increase sensitivity by converting to 'screen-detected' most interval cancers occurring in the second year, but this would not necessarily represent higher efficacy on account of many more false-positives and unnecessary colonoscopies. In any case, this is hardly a realistic option in Florence or in other European countries given limited resources.

Lowering the positivity threshold (e.g. to $70 \mathrm{ngml}^{-1}$ ) might have allowed the detection of two interval cancers with LAT between 70 and $100 \mathrm{ngml}^{-1}$ : if so, sensitivity would have been raised from 73.8 to $77.0 \%$ (or $75.4 \%$ with LAT between 80 and $99 \mathrm{ngml}^{-1}$ ). Unfortunately, our study could not assess the corresponding increase in advanced adenoma detection. On the other hand, lowering the positivity cutoff to 70 or to $80 \mathrm{ngml}^{-1}$ would have increased recall rate from 4 to $5.9 \%(1612 / 27503)$ or to $5.1 \%$ (1397/ 27 503), respectively.

Multiple FOBT testing of $2-3$ bowel movements has been proposed as another possible option to increase sensitivity, and 2-day testing has been suggested as the most cost-effective choice (Nakama et al, 1999; Yamamoto and Nakama, 2000). Lowering the positivity cutoff and doubling the number of sampled bowel movements, alone or combined, are presently being evaluated to increase screening accuracy in a multicentre study in Italy.

\section{ACKNOWLEDGEMENTS}

We are grateful to Antonio Gasparrini for his statistical assistance.
Clayton D, Hills M (1993) Statistical models in epidemiology. Oxford University Press: Oxford

Day NE (1985) Estimating the sensitivity of a screening test. J Epidemiol Community Health 39: $364-366$

Edwards JB (2005) Screening for colorectal cancer using faecal blood testing: varying the positive cut-off value (letter). Pathology 37: $565-568$

Grazzini G, Castiglione G, Ciabattoni C, Franceschini F, Giorgi D, Gozzi S, Mantellini P, Lopane P, Perco M, Rubeca T, Salvadori P, Visioli CB, Zappa M (2004) Colorectal cancer screening programme by faecal occult blood in Tuscany: first round results. Eur J Cancer Prev 13: 19-26

Guittet I, Bouvier V, Mariotte N, Vallee JP, Arséne D, Boutreoux S, Tichet J, Launoy J (2007) Comparison of a guaiac based and an immunochemical 
faecal occult blood test in screening for colorectal cancer in general average risk population. Gut 36: $210-214$

Itoh M, Takahashi K, Nishida H, Sakagami H, Okubo T (1996) Estimation of the optimal cut-off point in a new immunological faecal occult blood test in a corporate colorectal cancer screening programme. J Med Screen 3: $66-71$

Jensen BM, Kronborg O, Fenger C (1992) Interval cancers in screening with fecal occult blood test for colorectal cancer. Scand J Gastroenterol 27: $779-782$

Jouve JL, Remontet L, Dancourt V, Lejeune C, Benhamiche AM, Faivre J, Esteve J (2001) Estimation of screening test (Hemoccult) sensitivity in colorectal cancer mass screening. Br I Cancer 84: 1477-1481

Launoy G, Smith TC, Duffy SW, Bouvier C (1997) Colorectal cancer mass screening: estimate of faecal occult blood test sensitivity taking into account mean sojourn time. Int J Cancer 73: 220-224

Launoy GD, Bertrand HJ, Berchi C, Talbourdet VY, Guizard AVN, Bouvier VM, Caces ER (2005) Evaluation of an immunochemical fecal occult blood test with automated reading in screening for colorectal cancer in general average-risk population. Int J Cancer 115: 493-496

Levi Z, Hazazi R, Rozen P, Vilkin A, Waked A, Niv Y (2006) A quantitative immunochemical faecal occult blood test is more efficient for detecting significant colorectal neoplasia than a sensitive guaiac test. Aliment Pharmacol Ther 23: 1359-1364

Morikawa T, Kato J, Yamaji Y, Wada R, Mitsushima T, Shiratori Y (2005) A comparison of the immunochemical fecal occult blood test and total colonoscopy in the asymptomatic population. Gastroenterology 129: $422-428$

Moss SM, Hardcastle JD, Coleman DA, Robinson MH, Rodrigues VC (1999) Interval cancers in a randomized controlled trial of screening for colorectal cancer using a faecal occult blood test. Int J Epidemiol 28: $386-390$

Nakama H, Kamijo N, Abdul Fattah AS, Zhang B (1996) Validity of immunochemical faecal occult blood screening for colorectal cancer: a follow-up study. J Med Screen 3: 63-65

Nakama H, Yamamoto M, Kamijo N, Li T, Wei N, Fattah AS, Zhang B (1999) Colonoscopic evaluation of immunochemical fecal occult blood test for detection of colorectal neoplasia. Hepatogastroenterology 46: $228-231$
Nakama H, Zhang B, Zhang Y (2001) Evaluation of the optimum cut-off point in immunochemical occult blood testing in screening for colorectal cancer. Eur J Cancer 37: 398-401

Petrelli N, Michalek AM, Freedman A, Baroni M, Mink I, Rodriguez-Bigas M (1994) Immunochemical vs guaiac occult blood stool test: results of a community-based screening program. Surgical Oncology 3: 27-36

Saito H, Soma Y, Nakajma M, Koeda J, Tanaka S, Nakagawa K, Teranishi T, Matsuhe R, Uchida K, Morikawa H (2000) A case-control study evaluating occult blood screening for colorectal cancer with Hemoccult test and an immunochemical hemagglutination test. Oncol Rep 7: 815-819

Towler BP, Irwig L, Glasziou P, Weller D, Kewenter J (2000) Screening for colorectal cancer using the faecal occult blood test, hemoccult. Cochrane Database System Rev 2: CD001216

Vilkin A, Rozen P, Levi Z, Waked A, Maoz E, Birkenfeld S, Niv Y (2005) Performance characteristics and evaluation of an automated-developed and quantitative immunochemical, fecal occult blood screening test. Am J Gastroenterol 100: 2519-2525

Yamamoto M, Nakama H (2000) Cost-effectiveness analysis of immunochemical occult blood screening for colorectal cancer among three fecal sampling methods. Hepatogastroenterology 47: 396-399

Yamamoto Y, Hino M, Minami Y, Matsuo S, Takahashi H (1990) Quantitative assay for fecal hemoglobin using an occult blood immunoanalyser (OC-Sensor). J Clini Lab Instrum Reagents 13: 683-689

Yoshinaga M, Motomura S, Takeda H, Yanagisawa Z, Ikeda K (1995) Evaluation of the sensitivity of an immunochemical fecal occult blood test for colorectal neoplasia. Am J Gastroenterol 90: 1076-1079

Zappa M, Castiglione G, Giorgi D, Grazzini G, Paci E, Ciatto S (1998) Re: participation in colorectal cancer screening: a review (correspondence). JNCI 90: 465

Zappa M, Castiglione G, Grazzini G, Falini P, Giorgi D, Paci E, Ciatto S (1997) effect of faecal occult blood testing on colorectal cancer mortality. Results of a population-based case-control study in the district of Florence. Int J cancer 73: $2208-2210$

Zappa M, Castiglione G, Paci E, Grazzini G, Rubeca T, Turco P, Crocetti E, Ciatto $S$ (2001) Measuring interval cancers in population-based screening using different assays of fecal occult blood testing: The District of Florence experience. Int J Cancer 92: 151-154 